\title{
Improved Novel Routing Algorithm for Congestion Control in Wireless Sensor Network
}

\author{
Harwinder Singh Sohal \\ Assistant Professor, IT Deptt \\ LLRIET, Moga
}

\author{
Mandeep Kaur \\ Assistant Professor, ECE Deptt \\ LLRIET, Moga
}

\begin{abstract}
This paper is based upon the concept of improving the novel congestion control scheme in Wireless Sensor Network. In WSN Congestion is an important issue. Congestion occurs at the transport layer of the OSI model. The Novel Congestion Control Scheme proposed a rate-based Fairness-Aware Congestion Control Protocol (FACC), which controls congestion and achieves approximately fair bandwidth allocation for different flows. Congestion occurs at the intermediate nodes in WSN. This means the time during when intermediate nodes send Flow Control Acknowledgement to Source Node to describe the congestion status so as to control its flow rate will result in large amount of packet loss. This motivates the need of new technique to control the packet dropping at the intermediate nodes. So to avoid the packet dropping the proposed work introduced the Improved Novel Routing Algorithm (INOVEL) for Congestion Control in Wireless Sensor Network which works in three phases. These three phases plays an important role in utilizing the available free spaces of the neighbored nodes and free routing paths to accommodate the load of Congestion Affected Node's. This Routing Algorithm is implemented in NS-2 where the results show that such an algorithm improves the performance, efficiency of the network by decreasing end to end delay and increasing both the throughput and packet delivery fraction among the nodes.
\end{abstract}

\section{Keywords}

NOVEL, INOVEL, CAN

\section{INTRODUCTION}

A wireless sensor network (WSN) consists of spatially distributed autonomous sensor nodes to cooperatively monitor physical or environmental conditions such as temperature, sound, vibration, pressure, motion or pollutants. The developments of wireless sensor networks was motivated by military applications such as battlefield surveillance and are now used in many industrial and civilian application areas including industrial process monitoring, control machine, health monitoring environment, habitat monitoring, healthcare applications, home automation and traffic control [1]. The wireless sensor network is a temporary network in which there are no wires, no communication infrastructure and no administrative involvement. In such environment, it is necessary for the mobile computers to take help from other computers in forwarding a packet to the destination due to the limited range of each mobile host's wireless transmission. The characteristics of wireless sensor network are: limited energy, dynamic network topology, lower power, node failure; short range broadcast communication and multihop routing. Multihop routing is a significant service required for wireless sensor network. Internet and MANET (Mobile Wireless sensor network) routing techniques does not perform well in
WSN (Wireless Sensor Network) [2]. There are several key issues in wireless sensor network routing including:

1. Reliability: When the message travel among multiple nodes then it is important to have high reliability on each link otherwise the probability of message transferring the whole network would be low.

2. Integration with wake/sleep schedules: - To save power many nodes are kept into sleep state by wireless sensor network. The awake node does not choose an asleep node as the next node.

3. Real-Time:- In some applications the messages must arrive at a destination by a deadline. Due to the high degree of uncertainty in WSN it is difficult to develop routing algorithms with any guarantees. Protocols such as SPEED (Stateless protocol) and RAP (Route access protocol) use a notion of velocity to prioritize packet transmissions.

4. Mobility: - Routing becomes complicated if either source or destination or both are moving. This can be resolve by continuously updating local neighbour tables or by indentifying proxy nodes that help in keeping track of where the nodes are.

5. Security: - If conflict occurs, it can perpetrate a wide range of attacks on the routing algorithm which include black hole, selective forwarding, wormhole and denial of service attack.

6. Congestion: - Many WSN have regular or infrequent traffic. Congestion is a problem for more demanding WSN and is an issue with larger systems that include audio, video and having multiple base stations. Congestion can occur in wired as well as wireless sensor network. Wireless Sensor Network has an event driven [3] nature which leads to unpredictable network load. Typically, Wireless Sensor Network operates under idle or light load and then suddenly become active in response to a detected event. When the events have been detected the information transit is of great importance. However the burst traffic resulted from the detected events can easily cause congestion in the networks especially in high-rate applications. In Wireless Sensor Network when data converge towards the sink i.e., the base station congestion is more likely to happen at sensors which receive more data than they can forward. Basically congestion in Wireless Sensor Network occurs at intermediate nodes because at the intermediate nodes the incoming traffic (received and generated) exceeds the capacity of the outgoing link or link bandwidth. Congestion at intermediate nodes is shown in the following Figure 1. 


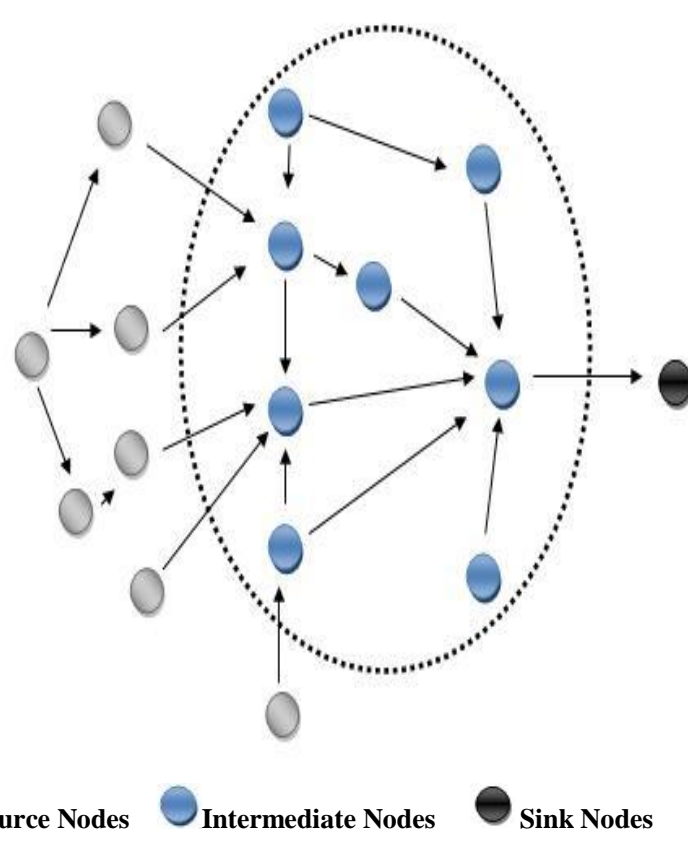

Figure 1: Congestion at intermediate nodes

Congestion in Wireless Sensor Networks (WSN) can be a severe problem [4] as it causes a plethora of malfunctions such as lower throughput, energy inefficiency and short lifetime. As a result, the performance of the whole network is subject to undesirable change an issue that renders congestion controls an issue of uttermost importance for such networks. Congestion also causes packet loss which in turn drastically decreases network performance and throughput. As sensors are energy constraint so it is a decisive task to detect the congestion and congested regions in a network to perform congestion control. In addition to that different application i.e. real time and non-real time data in sensor network have different QoS (delay, link utilization and packet loss) guarantee requirement.

\section{LITERATURE REVIEW}

The Proposed Work involves the introduction of Improved Novel Routing Algorithm for Congestion Control in Wireless Sensor Network. The available literature has been reviewed in this context.

Ying Ouyang, Chuang Lin, Fengyuan Ren, Hongkun Yang, Xiaomeng Huang [5] described that Backpressure (BP) under Active Network architecture is used to make congestion control more responsive to detect/recover congestion in WSN. They designed a simple Active Backpressure mechanism to allocate bandwidth Proportional to the Size of tree (ABPS) and they presented a fluid-based analytical model of ABPS using stochastic differential equations. ABPS introduced programs in each data packet that tell nodes how to react to congestion and quickly converge to a fair and efficient rate. They demonstrated a deterministic approach to analyze the stochastic model, in which they obtained a set of ordinary differential equations from their model and they derived the average behaviour of queue length and flow throughput from the ordinary differential equations. Finally, they evaluated ABPS extensively on a 50-node wireless sensor network. Simulation results validated the effectiveness of ABPS and match well with the theoretic analysis.
Yunlu Liu Yuheng Liu Juhua Pu Zhang Xiong [6] proposed a robust routing algorithm with fair congestion control in wireless sensor network (called RRAFCC). RRAFCC performs a low cost and robust routing based on dividing the monitoring region into several subareas initially. Furthermore, RRA-FCC provides a fair congestion control which improved the whole network fairness in congestion. The simulation results showed that RRA-FCC performs well in the throughput of Sink nodes, energy consumption and the whole network fairness in congestion.

Yuan Chen, Lemin Li [7] introduced that buffer management strategy in wireless networks can manage the buffer efficiently, avoid or relieve congestion so as to guarantee QoS. Because of unique issues in the wireless channel, strategies in wired networks can not apply directly to wireless network. In this paper they proposed a fair dropping algorithm called DS-CFD to decide drop policy in wireless networks when congestion occurs. The algorithm considered both channel condition and fairness so as to achieve tradeoffs between throughput and fair services and they give different level of balance to assured services and best effort services. In this paper they defined some another parameter is to modify resource allocation between different classes. That parameter was also adaptive with network load. They build a simple model of the algorithm and presented simulation results to show the performance improvements.

Can Basaran, Kyoung-Don Kang, Mehmet H. Suzer[8] presented a lightweight distributed congestion control method in WSNs. They developed a new metrics to detect congestion in each node by considering the queue lengths and channel conditions observed in the one hop neighbourhood. Based on the estimated level of congestion, each node dynamically adapts its packet transmission rate and balances the load among the one-hop neighbours to avoid creating congestion and bottleneck nodes.

Shigang Chen and Na Yang [9] proposed a congestionavoidance scheme based on lightweight buffer management. They described simple yet effective approaches that prevent data packets from overflowing the buffer space of the intermediate sensors. These approaches automatically adapt the sensors' forwarding rates to nearly optimal without causing congestion. They discussed how to implement bufferbased congestion avoidance with different MAC protocols. In particular for CSMA with implicit ACK our 1/k-buffer solution prevents hidden terminals from causing congestion. They demonstrated how to maintain near optimal throughput with a small buffer at each sensor and how to achieve congestion-free load balancing when there are multiple routing paths toward multiple sinks.

Hua-Feng Deng, Yun-Sheng Liu, Ying-Yuan Xiao [10] proposed a novel algorithm for load balancing in the parallel and distributed systems. Firstly, almost all jobs are organized into the standard job combinations, each of which consists of one to four jobs. Then they are assigned to the machines according to the assignment algorithm for job combinations, which was a special integer partition algorithm. The maximum error between the optimal algorithm and their algorithm was estimated. The experimental results showed that their algorithm performs efficiently.

Jang-Ping Sheu and Wei-Kai Hu [11] proposed a hybrid congestion control protocol that considered not only the packets delivery rate but also retained the buffer size of each 
node. Their proposed protocol may avoid packets drop due to traffic congestion and improve the network throughput. HCCP detects the congestion in advance with a time period $T$ and takes the preventive measures.

Minho Choi, Junhyung Kim, Sooyeol Yang, Namkoo Ha and Kijun Han [12] introduced that Congestion is generated by buffer overflow and generally considered in transport layer. However, there is almost no considered one about existing routing protocols of sensor network because it is difficult to apply congestion control in transport layer of WSNs (wireless sensor networks) that have limited resources such as energy, buffer and bandwidth of sensor nodes. Therefore, routing protocol of sensor networks must deliberate how to control congestion. In order to overcome this problem they proposed the routing protocol avoidable congestion to improve throughput, packet drop rate, life time and so on. Consequently each node can evenly distribute loads. They defined how to achieve congestion-free load balancing when there are multiple routing paths towards sink.

\section{PRESENT WORK}

In WSN data has to pass through many intermediate nodes when it travels from Source node to Sink node. The event driven nature of WSN leads it to unpredictable network load as a result congestion occur at the nodes (i.e. intermediate nodes) which receives more data than they can forward and cause packet losses. The reason behind the occurrence of Congestion is that if for any time interval the total sum of demands on a source is more than its available capacity then for that time interval the source is said to be congested. Congestion has dreadful consequences in terms of network utilization, energy efficiency and packet loss in WSNs. The level of congestion is directly proportional to the dropping probability of packets. As congestion increases more \& more packets get dropped and the throughput significantly decreases. Moreover, when a packet is dropped the energy spent by upstream sensors on the packet is wasted. The farther the packet has traveled the greater the waste is. Packets dropped at intermediate nodes must be retransmitted, but low bandwidth of WSN does not allow this, also cause delay in data transmission. So it becomes necessary to protect those packets bounded to be dropped.

\section{OBJECTIVES}

The objectives of Improved Novel Routing Algorithm in Wireless Sensor Network are as follows:

1. To make the Maximum use of free nodes and their buffer space.

2. To reduce End to End Delay.

3. To increase the Packet Delivery Fraction.

4. To achieve the maximum Throughput.

\section{METHODOLOGY}

The Proposed Algorithm Works in three Phases. The first phase is query forwarding phase. The next phase is exchanging control messages between parent node and child nodes. Last one is routing including residual buffer sizes of neighbours.

\subsection{Phase 1}

Firstly, Congestion Affected Nodes (CAN) will broadcast indication messages to all the neighbouring nodes. After receiving the messages, the neighbouring nodes calculate the buffering queue length by implementing RED algorithm, put the results into the header of message and send it back to the congestion affected nodes so that the congestion affected nodes can grasp the congestion information regarding the buffer length of the neighbouring nodes and then create the table of which contain the information regarding congestion. Figure $2 \& 3$ shows that how Congestion Affected Nodes are broadcasting indication messages to the Neighboring Nodes.

\section{Broadcast transmission}

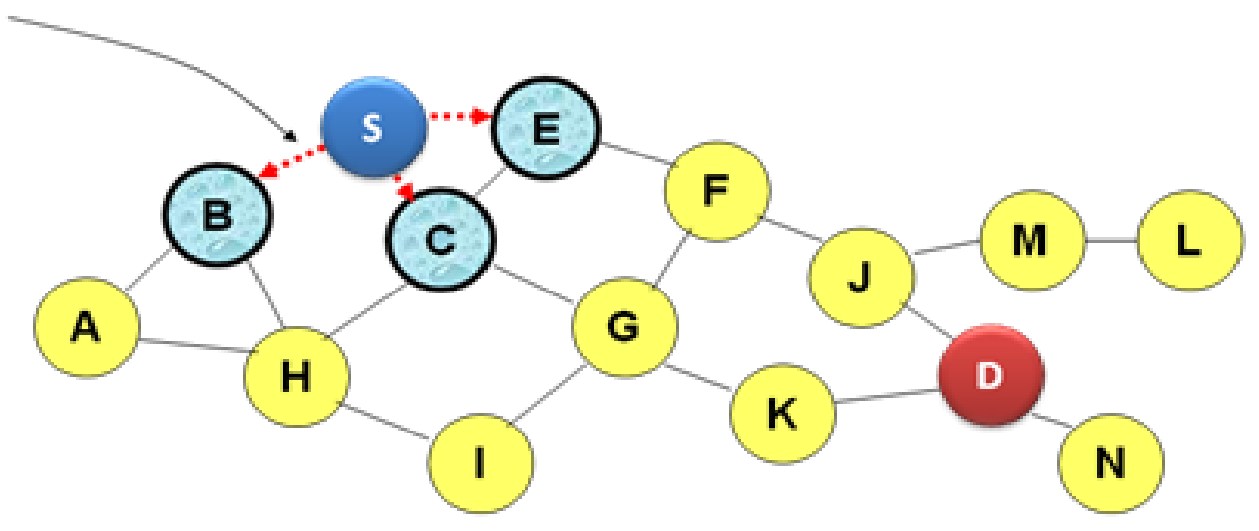

\section{Represents transmission of Indication Message}

Figure 2: Congestion Affected Node's are broadcasting indication messages 


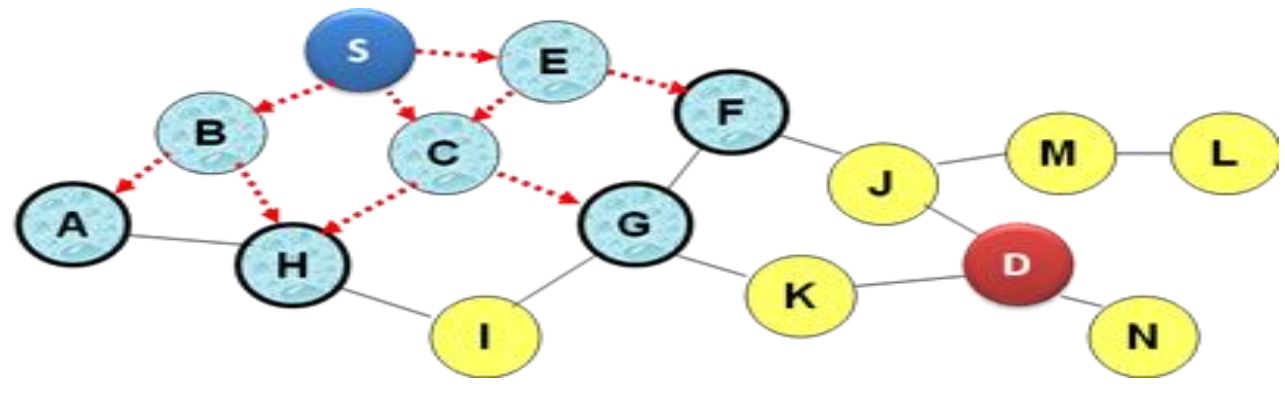

Figure 3: Congestion Affected Node's are broadcasting indication messages to each neighboring nodes

As soon as each neighbour node receives the indication message from the CAN each node implements RED algorithm. The RED algorithm can control the buffering queue length of the router to a relative lower value, receive the data packets arrived instantly and prevent data packets from the burst loss so that it increases the usage of the bandwidth. Following are the steps of RED algorithm to calculate the buffer length of node.

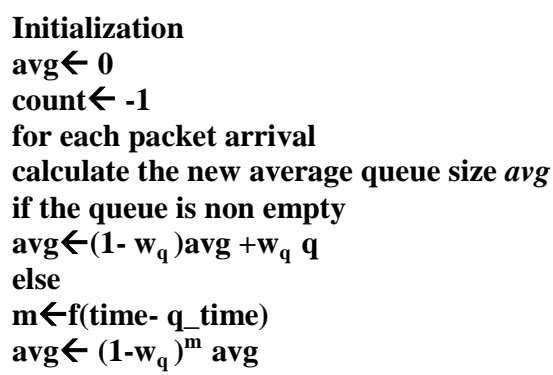

The RED gateway computes the average queue size at packet arrivals rather than fixed time intervals, the calculation of the average queue size is modified when a packet arrives at the gateway to an empty queue. After the packet arrives at the gateway to an empty queue, the gateway calculates $\boldsymbol{m}$. the number of packets that might have been transmitted by the gateway during the time that the line was free. The gateway calculates the average queue size as if $\boldsymbol{m}$, packets had arrived at the gateway with a queue size of zero. The calculation is as follows:

$$
\begin{aligned}
& \mathrm{m} \leftarrow \mathrm{f}\left(\text { time- } \mathrm{q}_{-} \text {time }\right) \\
& \operatorname{avg} \leftarrow\left(1-w_{\mathrm{q}}\right)^{\mathrm{m}} \text { avg }
\end{aligned}
$$

Where $q_{-}$time is the start of the queue idle time and $s$ is a typical transmission time for a small packet. This entire calculation is an approximation, as it is based on the number of packets that might have arrived at the gateway during a certain period of time. The RED at neighbor node uses a low pass filter to calculate the average queue size. The low pass filter is an exponential weighted moving average (EWMA).

$$
\operatorname{avg} \leftarrow\left(1-w_{q}\right) \operatorname{avg}+w_{q} q
$$

Where the weight $\mathbf{w}_{\mathbf{q}}$ determines the time constant of the low pass filter. On the other hand when the packet arrives at the neighbor node to an empty queue, the node calculates $\mathrm{m}$, the number of packets that might have been transmitted by the CAN during the time that the line was free. The neighbor node calculates the average queue size as if $\mathrm{m}$, packets had arrived at the gateway with a queue size of zero. Where q-time is the start of the queue idle time and $s$ is a typical transmission time for a small packet. This entire calculation is an approximation, as it is based on the number of packets that might have arrived at the neighbor node during certain period of time.

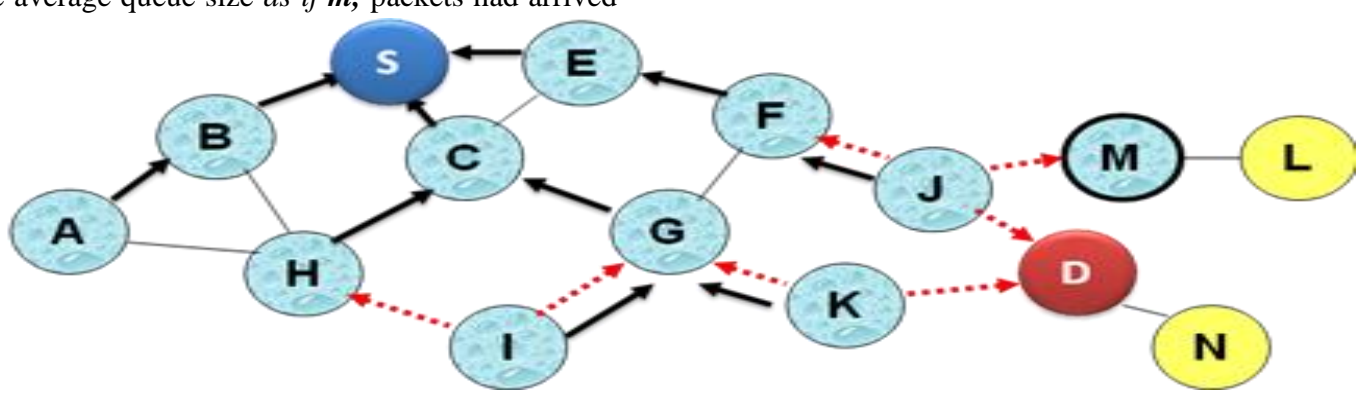

Figure 4: Each neighboring node after calculated buffer length is unicasting back to the CAN

\subsection{Phase 2}

In congestion information table, each record has an updatetime field to represent whether this record is latest or not. Compared the update time with a fixed threshold value, if it is less than threshold value, it means that record is latest and effective. If not, it means the record has been obsolete. Before they transmit the data, congestion affected nodes first inquire the congestion information table whether the records are new or not. If not, active nodes again send indication message immediately to the neighbouring nodes to update the records, Also RED algorithm implements the piggybacking procedure to update queue length in the neighbour table. After updating the table of neighbour nodes CAN find out those neighbouring nodes whose buffer space is maximum. It indicates that the network resource of this node is most sufficient and this node is the right one that data packets are sent to. 


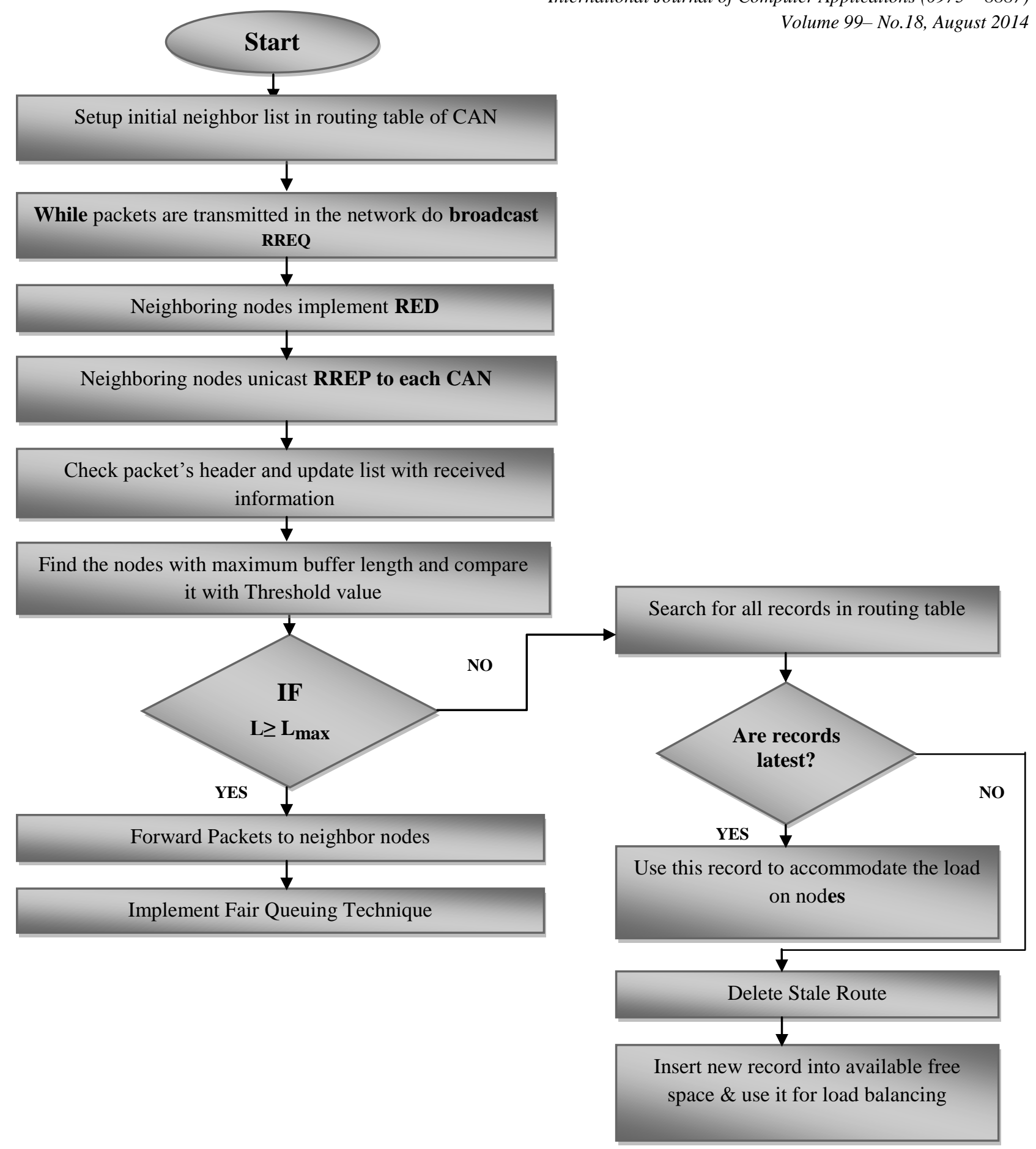

Figure 5: Flow Chart of Novel Routing Algorithm

\subsection{Phase 3}

After finding the nodes with maximum buffer length routing is done. Routing in wireless sensor networks has focused on energy efficiency as a key design metric. The transport layer should work in sync with the Novel Routing Algorithm to enable maximum conservation of the limited energy. In WSN Multipath routing technique is used i.e. there are multiple paths from source to destination to effectively route the packet. Multipath routing technique is used so that if a single link fails, there is always a secondary route available to reroute the packets. This characteristic of multiple routes could also be used to implement congestion avoidance by re-routing packets when neighbour nodes' queues are gradually filling up. In WSN a packet drop on a broken link has the same effect as a packet drop due to buffer overflow. In such a situation, if multiple routes are available to the Source it could make a routing decision to route its packet through a different node. This method of functioning simultaneously results in a self-regulating system of fairness and load balancing in the network. Packets are forwarded to nodes where the chances of packet drop due to buffer overflow is minimum and coupled with fair queuing techniques to achieve higher degree of packet delivery fraction in the WSN. 


\section{SIMULATION SETTINGS}

The simulation experiment is carried out in LINUX (FEDORA 10). The detailed simulation model is based on network simulator-2 (ver-2.34) is used in the evaluation. The NS instructions can be used to define the topology structure of the network and the motion mode of the nodes, to configure the service source and the receiver, to create the statistical data track file and so on.

\subsection{Mobility Model}

Mobility model represents the movement of mobile users and how their location, velocity and acceleration change over time. Such models are frequently used for simulation purpose when new communication or navigation techniques are investigated. The mobility model uses the random waypoint model in a rectangular field. The field configurations used is: $500 \mathrm{~m} \times 500 \mathrm{~m}$ field with 50 nodes, maximum speed of 20 $\mathrm{m} / \mathrm{sand}$ simulation time of $100 \mathrm{sec}$ 's.

\subsection{Simulation Results}

The result is simulated is using NS-2.34 software by taking the parameter Pause Time on X-axis and performance metrics (End To End Delay, Packet Delivery Fraction \& Throughput ) on Y-axis. Pause time is the idle time for which the mobile nodes in wireless sensor network are in stable position. In the following graphs red line indicates the INOVEL \& green line indicates the NOVEL.

\section{i. End To End Delay \& Pause Time}

End to End delay represents the time when packet was received at destination minus the time when packet was created by the source divided by the Number of packet delivered at destination .In comparing the end-to-end delay, INOVEL is better as compared to NOVEL. As shown in the graphical representation in figure 6 that the end to end delay in INOVEL is lesser than NOVEL. With the decrease in end to end delay the network's lifetime \& performance is improved.

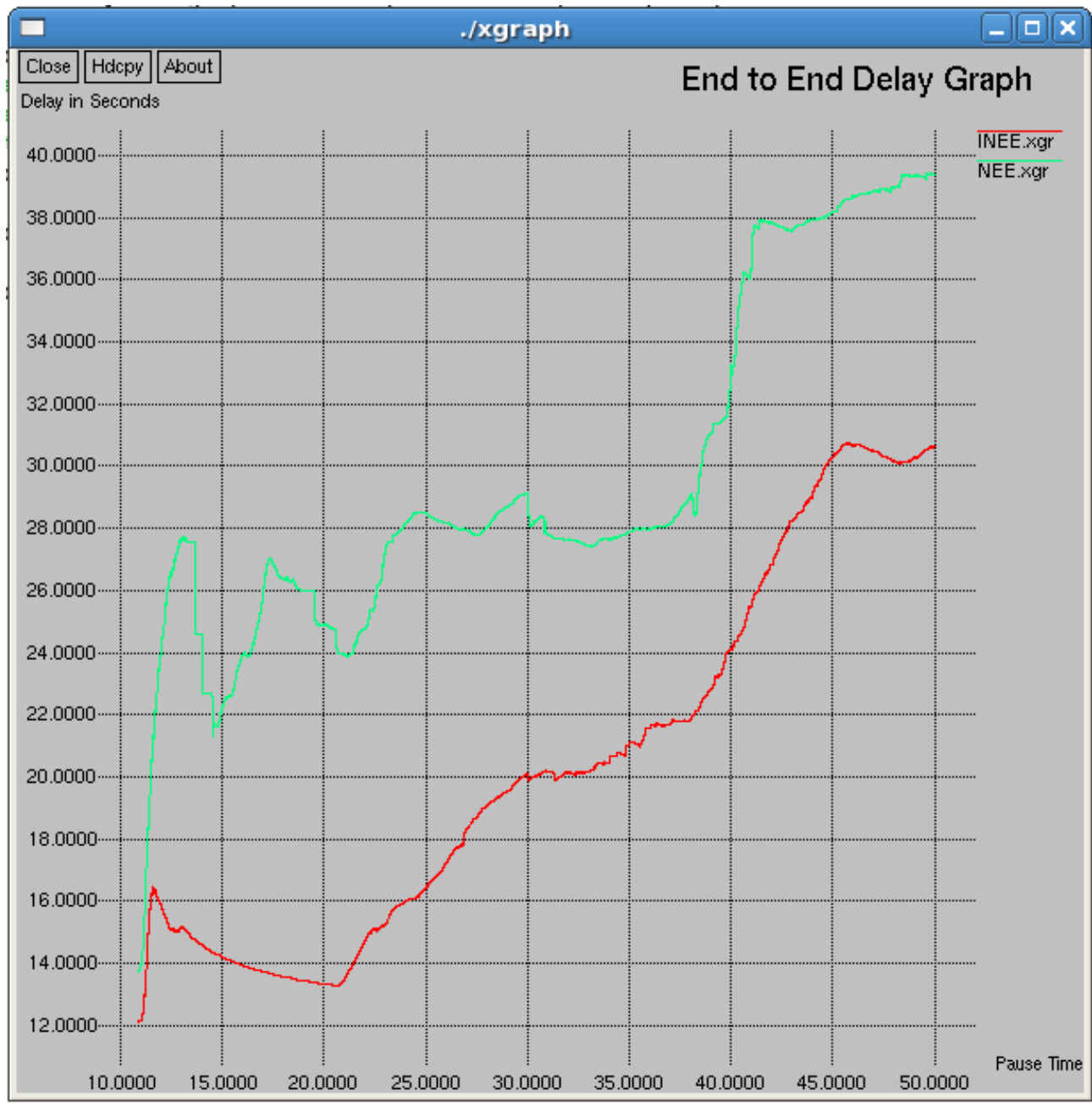

Figure 6: Average End-End Packet Delivery \& Pause Time Graph of INOVEL \& NOVEL

\section{ii. Packet Delivery Fraction \& Pause Time}

The packet delivery fraction represents the ratio of the number of packet generated at the source to the number of packets delivered by the destination. It specifies the packet loss rate, which limits the maximum throughput of the network. The better the delivery ratio, the more complete and correct the routing protocol. Following figure 7 represents that the packet delivery fraction of INOVEL is better than NOVEL and is improved by balancing the network load. 


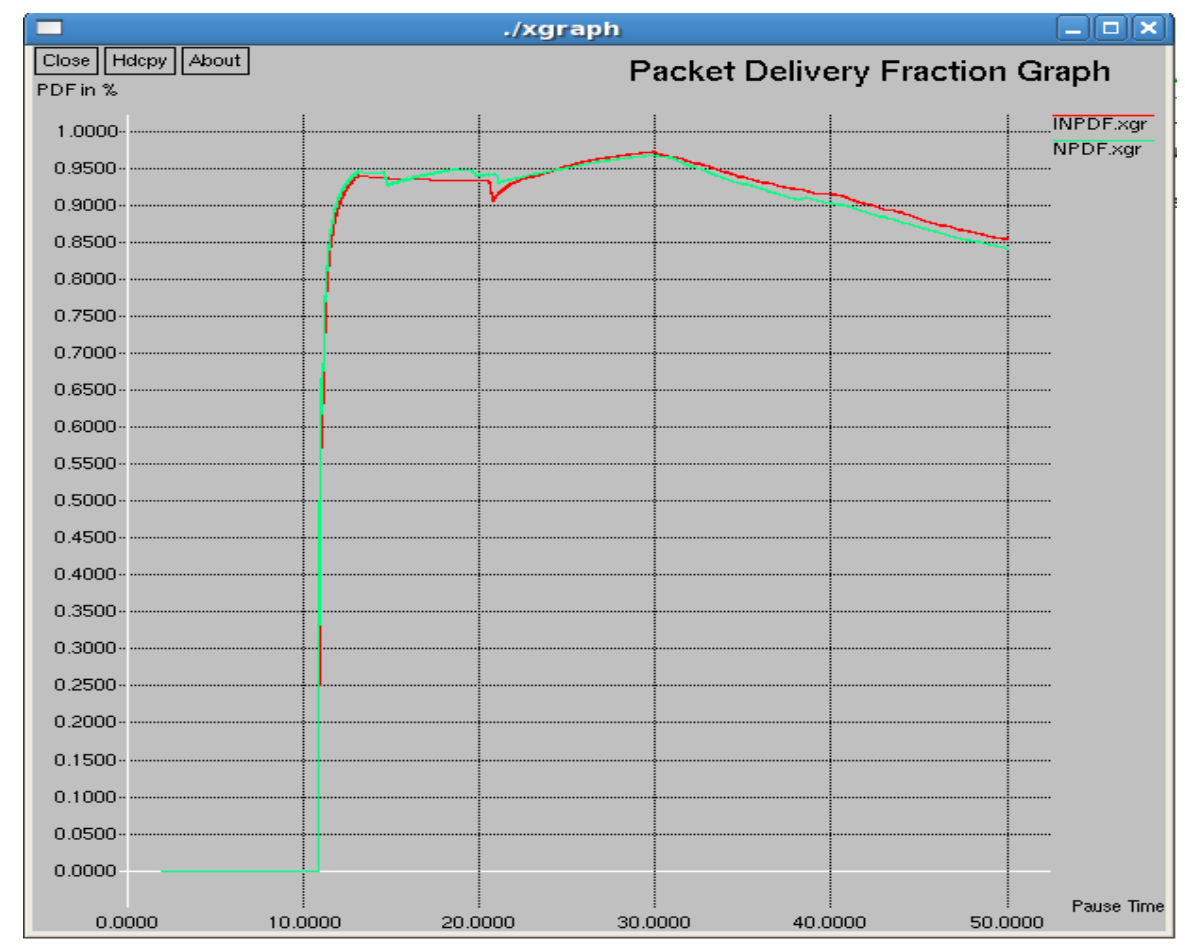

Figure 7: Packet Delivery Fraction \& Pause Time Graph of INOVEL \& NOVEL

\section{iii. Throughput \& Pause Time}

Throughput is the rate of successful delivery of data over a communication channel. Throughput metric represents the number of packets delivered at each node in the network over the time interval taken due packets delivery. The throughput is usually measured in data packets per second or data packets per slot. Throughput is essentially synonymous to digital bandwidth consumption. As illustrated in Figure 8 the throughput comparison of NOVEL \& INOVEL. NOVEL demonstrates significantly lower routing load than INOVEL, with the factor increasing with a growing number of sources.

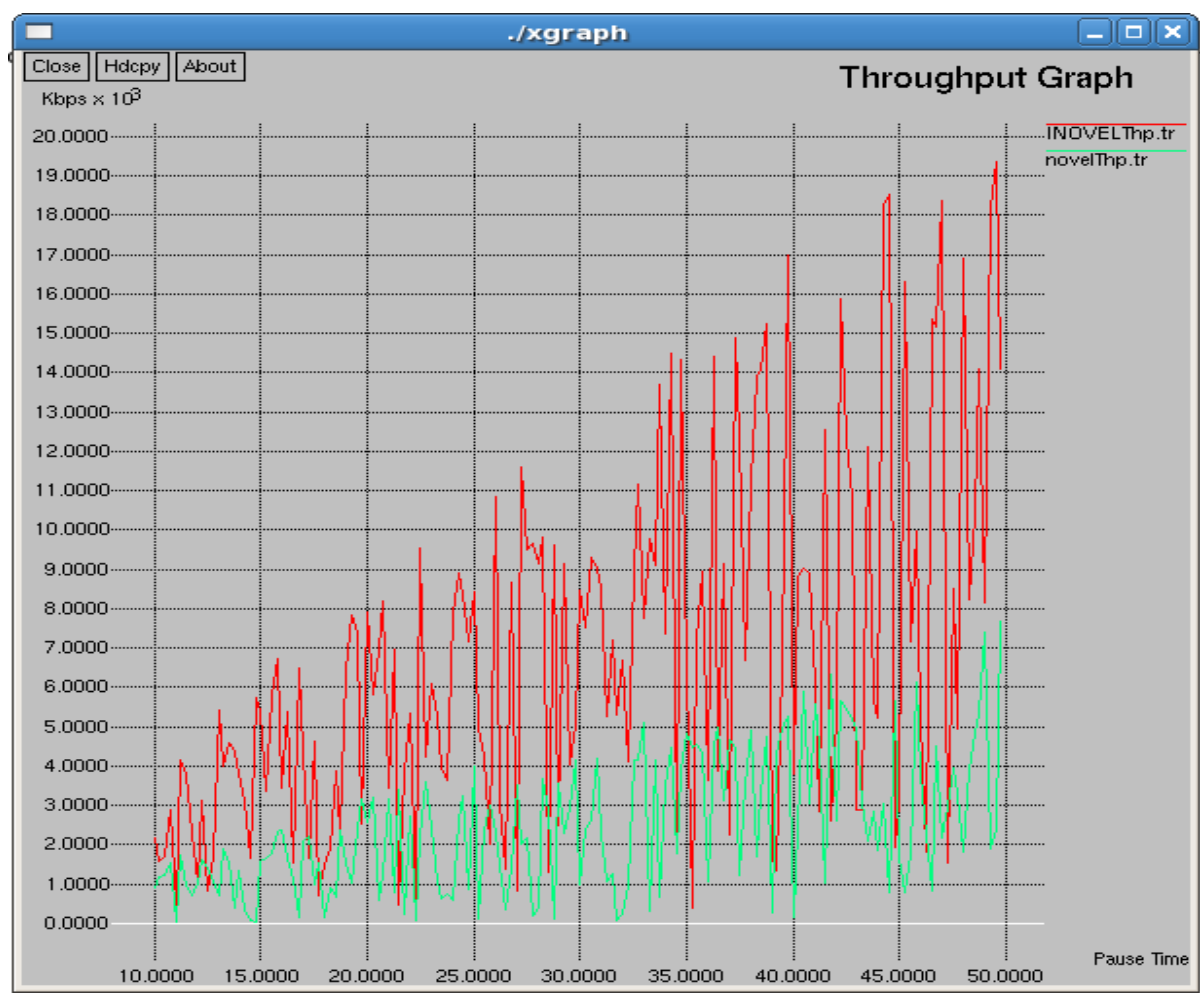

Figure 8: Throughput \& Pause Time \& Pause Time Graph of INOVEL \& NOVEL 


\section{iv. Performance Comparison of both NOVEL and INOVEL}

Following table shows the performance of the both the Algorithms with respect to the difference metrics

Table 1: Performance Comparison of NOVEL and INOVEL

\begin{tabular}{|c|c|c|}
\hline $\begin{array}{c}\text { Performance } \\
\text { Parameters }\end{array}$ & NOVEL & INOVEL \\
\hline End-to-end delay & High & Low \\
\hline Packet delivery ratio & Low & High \\
\hline Throughput & Low & High \\
\hline $\begin{array}{c}\text { No. of Paths for } \\
\text { Routing }\end{array}$ & Single & Multipath \\
\hline
\end{tabular}

\section{CONCLUSIONS \& FUTURE WORK}

In the proposed work an improved novel routing algorithm is introduced by using the concept of buffer length. The buffer length of each neighboring node is calculated by RED Algorithm \& is used to make routing decision to increase the packet delivery fraction at intermediate nodes for the purpose of reducing congestion. The Algorithm in proposed work achieves high performance efficiently in a very innovative way. Also, this algorithm is used at the transport layer for reporting events helps to avoid packet drops in dense regions and increases the packet delivery ratio at the destination. This proposed work is efficient for using the network resources, provide reliable data transmission and reduces the network overhead. The simulation results demonstrated that the during the congestion the intermediate nodes consumes the free available buffer length of neighbor nodes to decrease the End to End Delay and to achieve high performance in terms of both Packet Delivery Fraction and Throughput. The higher Packet Delivery Ratios with lesser transmissions leading to an increase in the lifetime of the network.

In Future an improved novel routing algorithm may works in an isolated control hardware which will run parallel along with many processing units. The control hardware micro program runs multithreaded subprograms where each threaded process dynamically created corresponding to that incoming request to that node. Each threaded has its separate hardware buffer to handle the packets from a particular request address.

\section{REFERENCES}

[1] LIU Yong-Mi, JIANG Xin-Hua "A Extended DCCP Congestion Control in Wireless Sensor Networks". IEEE- 2009.
[2] Kumar, J. (2010), "Evaluation of Energy-Aware QoS Routing Protocol for Ad Hoc Wireless Sensor Networks", International Journal of Electrical and Electronics Engineering, Vol. 4, pp. 413- 416.

[3] Xiaoyan Yin, Xingshe Zhou, Rongsheng Huang, Yuguang Fang, and Shining Li 2009" A Fairness-Aware Congestion Control Scheme in Wireless Sensor Networks". 2009 Fifth International Conference on Mobile Ad-hoc and Sensor Networks.

[4] Vasilis Michopoulos, Lin Guan, Iain Phillips Department of Computer Science, Loughborough University, LE11 3TU, UK "A New Congestion Control Mechanism for WSNs" 2010 10th IEEE International Conference on Computer and Information Technology (CIT 2010).

[5] Ying Ouyang, Chuang Lin, Fengyuan Ren, Hongkun Yang, Xiaomeng Huang "Design and Analysis of a Backpressure Congestion Control Algorithm in Wireless Sensor Network".

[6] Yunlu Liu* Yuheng Liu Juhua Pu Zhang Xiong "A Robust Routing Algorithm with Fair CongestionControl in Wireless Sensor Network".

[7] Yuan Chen, Lemin Li "A Fair Packet Dropping Algorithm Considering Channel Condition in Diff-Serv Wireless Networks". Key Lab of Broadband Optical Fiber Transmission and Communication System Technology University of Electronic Science and Technology, Chengdu, 610054, China.

[8] Can Basaran, Kyoung-Don Kang, Mehmet H. Suzer "Hop-by-Hop Congestion Control and Load Balancing in Wireless Sensor Networks" 35th Annual IEEE Conference on Local Computer Networks LCN 2010, Denver, Colorado.

[9] Shigang Chen and Na Yang "Congestion Avoidance Based on Lightweight Buffer Management in Sensor Networks" IEEE TRANSACTIONS ON PARALLEL AND DISTRIBUTED SYSTEMS, VOL. 17, NO. 9, SEPTEMBER 2006

[10] Hua-Feng Deng, Yun-Sheng Liu, Ying-Yuan Xiao "A Novel Algorithm for Load Balancing in Distributed Systems" Eighth ACIS International Conference on Software Engineering, Artificial Intelligence, Networking and Parallel/Distributed Computing.

[11] Jang-Ping Sheu and Wei-Kai Hu "Hybrid Congestion Control Protocol in Wireless Sensor Networks" 2008.

[12] Minho Choi, Junhyung Kim, Sooyeol Yang, Namkoo Ha and Kijun Han "Load Balancing for Efficient Routing in Wireless Sensor Networks", 2008 International Multisymposiums on Computer and Computational Sciences. 